\title{
The Pictorial Attitude Implicit Association Test for Need for Affiliation
}

Hendrik Slabbinck, Jan De Houwer, and Patrick Van Kenhove

Ghent University, Belgium

Word count: 3822

In Press. Personality and Individual Differences.

\begin{abstract}
Author Note
Hendrik Slabbinck, Department of Marketing, Ghent University;

Jan De Houwer, Department of Psychology, Ghent University;

Patrick Van Kenhove, Department of Marketing, Ghent University.
\end{abstract}

Correspondence concerning this article should be addressed to Hendrik Slabbinck, Department

of Marketing, Ghent University, Tweekerkenstraat 2, B-9000 Ghent, Belgium.

Email: Hendrik.Slabbinck@UGent.be; Phone: 0032926435 67; Fax: 003292644279 


\section{Highlights}

- We validated the Pictorial Attitude-IAT (PA-IAT) as measure of need for affiliation

- The PA-IAT predicts non-declarative behavior if need for affiliation is activated

- The PA-IAT is not related to direct measures of need for affiliation 


\begin{abstract}
The Pictorial Attitude Implicit Association Test (PA-IAT) has recently been developed as a new measure of need for power. This study aims to extend the scope of the PA-IAT to need for affiliation. In line with implicit motive theory, results show that the need for affiliation PA-IAT predicts non-declarative measures of motivation provided that need for affiliation is activated. We also obtained evidence for the discriminant validity of the measure. In general, our results confirm that the PA-IAT is a valid measure of implicit motives and show that the PA-IAT can also be used for the assessment of need for affiliation.
\end{abstract}

Keywords: implicit motives, need for affiliation, Implicit Association Test (IAT) 


\section{The Pictorial Attitude Implicit Association Test for Need for Affiliation}

\section{Introduction}

Research on implicit motives hibernated for a long time, but today the interest in implicit motives resurged. Implicit motives are basic needs shared by all human beings. They are assumed to be derived from emotional experiences during prelingual socialization processes, to operate outside a person's consciousness and to orient, select, and energize behavior (McClelland, Koestner, \& Weinberger, 1989). Ample empirical evidence shows that implicit motives are affect based, preferentially aroused by nonverbal stimuli, and influence nondeclarative measures of motivation and behavior (e.g., implicit learning, physiological responses, nonverbal behavior). In this respect, implicit motives are the mirror image of explicit motives that are cognition based, preferentially aroused by verbal stimuli, and influence declarative measures of motivation and behavior (e.g., decisions, deliberative choices, attributions, ...) (Schultheiss, 2008, for an overview).

Implicit motive research is concentrated around the 'big three' implicit motives: need for power, need for achievement, and need for affiliation. Each motive represents an enduring preference for a specific class of affective incentives (Schultheiss, 2008). More precisely, power motivated people derive pleasure from having emotional or behavioral impact on others, and experience impact from others as aversive (Winter, 1973). People high in need for achievement get satisfaction from unique accomplishments, strive for successful attainment of standards of excellence, but feel disappointed with failure or lack of excellence (McClelland, 1985). Finally, people high in need for affiliation take pleasure in establishing, maintaining, or restoring a positive affective or intimate relation with another person or a group of people, and evaluate separation or disruption of friendly relationships as unpleasant (Schultheiss, 2008). 
Because implicit motives largely operate outside one's consciousness, they are best measured by indirect measures. The most popular class of indirect technique to assess implicit motives is the Picture Story Exercises (PSE: Schultheiss \& Pang, 2007). A typical PSE consists of four to six pictures that depict people in a variety of social settings. For each picture, participants are asked to write an imaginative story. Because these tests use non-verbal cues, they are assumed to arouse implicit motives (e.g., McClelland et al., 1989). The content of the stories can be coded according to motivational coding systems, empirically derived and refined over decades (Smith, 1992; Winter, 1994). Finally, the summed scores yield measures of a participant's implicit motives. Recent work confirms the good psychometric qualities of PSE measures (Schultheiss \& Pang, 2007). On the other hand, doubts about its validity persist. Moreover, the measure requires a labor-intensive administrative procedure, which has led researchers to develop alternative measurement techniques to assess implicit motives. However, attempts to replace content based scoring techniques with objective and easy-to-use questionnaire measures of implicit motives had limited success (see e.g. Schultheiss, Yankova, Dirlikov, \& Schad, 2009, for a detailed discussion) and, in fact, reinforced the premise that implicit and explicit motives can best be seen as distinct, but related constructs, each activated by different classes of incentive, and each influencing different types of behavior.

The rise of a new class of implicit measures that are based on response latencies offered new possibilities for the development of alternative implicit motive measures. The most interesting alternative to the PSE and other content-coding techniques is offered by variants of the Implicit Association Test (IAT: Greenwald, McGhee, \& Schwartz., 1998). Many researchers consider the IAT to be the most reliable indirect measure that is currently available (e.g. De Houwer, Teige-Mocigemba, Spruyt, \& Moors, 2009). Brunstein and Schmitt (2004) and 
Sheldon, King, Houser-Marko, Osbaldiston, and Gunz (2007) were the first to apply the Implicit Association Test to assess implicit motives. Recently, Slabbinck, De Houwer, and Van Kenhove (2011) optimized the properties of the IAT for the assessment of need for power. In short, they showed that an IAT that employed pictorial target stimuli and affective attribute categories correlated more strongly with non-IAT measures of implicit motives than other IAT variants that employed verbal target stimuli and/or attribute categories that referred to the self. They argued that the pictorial version of the IAT is superior to the other variants because (a) implicit motives are based on early, non-verbal experiences, which can best be aroused by pictorial stimuli (McClelland et al., 1989) and (b) words are more likely to elicit concept-irrelevant associations (e.g. the word 'power' might elicit associations that refer to 'electrical energy', or 'mathematical operation' too) (Gschwendner, Hofmann, \& Schmitt, 2008).In the Pictorial Attitude IAT (PAIAT) of Slabbinck et al. (2011), participants categorized six pictures featuring power situations (e.g., business man standing in front of his private jet) and six pictures featuring non-power situations (e.g., happy family walking hands in hands)according to the labels "Power" or "Not power". In addition, 6 positively valenced (e.g. lovely) and six negatively valenced words (e.g., annoying) were classified as referring to the labels "Attractive" or "Not attractive". In another study, Slabbinck et al. (in press) established convergent, discriminant and incremental validity of the PA-IAT vis-à-vis the PSE. In sum, the PA-IAT is a promising tool for the assessment of implicit motives, but up to now, only a version exists for the assessment of need for power.

The aim of the present study is to extend the scope of the PA-IAT to need for affiliation. This is important because a wider range of implicit motive measures could yield a better understanding of the underlying psychological constructs. Moreover, the availability of an easyto-use measure could stimulate further implicit motive research. Because the need for affiliation 
PA-IAT requires pictures that represent the need for affiliation concept either very well or not at all, we first conducted a pretest that focused on the selection of the pictorial stimuli. The main study deals with the predictive and discriminant validity of the need for affiliation PA-IAT. To test the predictive validity, we rely on the traditional model of implicit motives (McClelland et al., 1989) which stipulates that individual differences in implicit motives predict non-declarative behavior, provided that the underlying implicit motive is aroused (see also Schultheiss, 2008 for a detailed discussion of this model).We therefore first measured need for affiliation using the PA-IAT and then assessed relevant non-declarative behavior when need for affiliation was or was not activated. We aroused need for affiliation for half of the participants by preventing them from engaging in a social (computer) game. Previous research repeatedly showed that social exclusion activates needs for affiliation (Williams, 2007) which in its turn leads to an enhanced sensitivity to positive social cues (e.g., Pickett, Gardner, \& Knowles, 2004).The to-be-predicted non-declarative behavior was assessed in the following manner. We exposed participants to printed advertisements and asked them to describe these advertisements afterwards. All advertisements were pretested and had a strong pull on need for affiliation. That is, all advertisements comprised several codable cues of affiliation imagery (e.g., friends having a barbeque on the shores of a lake, two men and a woman talking in kitchen, ...). The descriptions were coded according to Winter's manual for scoring motive imagery in running text (Winter, 1994)and resulted in scores for need for affiliation, need for power, and need for achievement. We expected that participants with an activated need for affiliation orient their attention to motive congruent cues (McClelland et al., 1989) and thus, that the PA-IAT measure of need for affiliation would be correlated only with the number of need for affiliation related excerpts in their descriptions of the printed advertisements and not with the number of need for power and 
need for achievement related excerpts. Discriminant validity was assessed also by correlating the PA-IAT measure of need for affiliation with explicit motive measures. Relying on the basic premise that implicit and explicit motive measures are measures of distinct constructs (Schultheiss et al., 2009), we did not expect the PA-IAT to correlate with any explicit motive measure.

\section{Method}

\subsection{Pretest}

In line with Slabbinck et al. (2011), we collected a series of 50 pictures that represented situations where need for affiliation was either an important motive or not at all. Seventy-four undergraduate university students evaluated each picture on their fit with need for affiliation. Fit with need for affiliation was examined by means of four statements and were inspired by Schultheiss' (2008) definition of need for affiliation (see the Appendix for an overview of the statements). Participants rated the pictures on a 7-point Likert scale with the following anchor points: $1=$ "Fits very well with the description", through $7=$ "Fits not at all with the description". Individual measures were calculated as the mean score of the scale items. The seven pictures with the highest (Range of Means: 5.8 -6.2) and the seven pictures with the lowest mean scores(Range of Means: 1.9-3.2) were selected for usage as exemplars of the affiliation concept of the need for affiliation PA-IAT. Internal consistencies of ratings of the pictures were satisfactory $(\alpha>.70)$.

\subsection{Design and participants}

In the main study, we first assessed need for affiliation by means of the PA-IAT and an explicit measure. In addition, participants completed another explicit measure that yielded a 
relative score of all three motives. Afterwards, participants were exposed to an experimental manipulation in which need for affiliation was aroused for half of the participants. Finally, participants described five printed advertisements. Advertisements descriptions were used to construct the measure of non-declarative behavior.

One-hundred twenty-eight graduate and undergraduate students completed the study and received 7 Euros for participation. Eighty-one participants were women (63\%) and mean age was $25(\mathrm{SD}=10.63)$. Need for affiliation was aroused for 55 participants $(43 \%)$.

\subsection{Measures and procedures}

2.3.1. Need for affiliation: PA-IAT. We relied on the procedure of Slabbinck et al. (2011, in press) for the construction of the PA-IAT. That is, for the target categories, we used the labels "together" and "alone," whereas for the attribute categories, we used "attractive" versus "not attractive". The stimuli representing the target categories featured pictures that represented affiliation situations (e.g., kids walking hand in hand on beach) or non-affiliation situations (e.g. business man standing up straight at meeting table). For the attribute categories, we used the stimuli "nice," "friendly," "pleasant," "great," "lovely," and "decent” to designate attractive, whereas we included “creepy," "unpleasant," "nasty," “unfavorable," "annoying," and "undesired" to represent not attractive. Following Greenwald et al. (1998), our IAT consisted of seven blocks of trials. In Block 1 (24 trials) participants sorted "Attractive" and "Not attractive" related items into the "Attractive" and "Not attractive" categories. Block 2 (24 trials) required participants to distinguish items representing "Together" or "Alone”. Block 3 (24 trials) and Block 4 (40 trials) combined the "Attractive - Not attractive" categories and the "Together Alone" categories. Block 5 (24 trials) was identical to Block 2, except that the positions of the “Together" and "Alone" categories were reversed. Finally, Block 6 (24 trials) and Block 7 (40 
trials) were identical to Blocks 3 and 4 except for the reversed position of the "Together" and "Alone" categories. Data from all combined blocks were used to compute IAT scores (D1 measure: Greenwald, Nosek, \&Banaji, 2003).Scores were calculated such that a high (low) IAT score represents a strong (weak) implicit need for affiliation.

2.3.2. Need for affiliation: explicit measure. We adopted the affiliation subscale of the Personality Research Form (PRF: Jackson, 1984) to measure the explicit need for affiliation. The scale consists of 12 items (e.g. "I try to be in the company of friends as much as possible"). Participants indicated to what extent each statement fitted them on a 5-point Likert scale with the following anchor points: $1=$ "Does not fit at all" and $5=$ "Fits very well". After recoding the reversed items, an explicit affiliation motive score was computed by taking the average score of all items. Internal consistency was satisfactory ( $\alpha=.79, M=3.60, S D=.48)$.

2.3.3. Relative explicit measure of motives (Constant Sum Scale).We used a relative explicit motive measure as previously described by Slabbinck et al. (2011). For this, participants were asked to distribute exactly 100 points among need for power, need for affiliation and need for achievement. After reading a short description of each motive, respondents indicated to what extent each motive fitted them by means of allocating a portion of 100 points to these motives. More points indicated a better fit.

2.3.4. Experimental manipulation. In order to arouse need for affiliation, we used Cyberball (Williams, 2007). Cyberball is a virtual ball-toss game that simulates social inclusion or exclusion and is regularly used to activate need for affiliation (Williams, 2007). Participants were told they would be playing a computerized ball-toss game via the Internet against two other players who were said to be participating in the same experiment in another PC lab. They were also told that they would get a message on their screen once their opponents were ready to start 
the game. The two other players were actually computerized, virtual players and were represented on the computer screen by a smiley and a nickname, randomly drawn from lists of 10 smileys and nicknames. Participants could choose their own nickname and got the opportunity to select a smiley from the same list from which the two other players got their smiley. At the start of the game, one of the virtual players got the ball and tossed the ball to one of the other players. Upon receipt of the ball, participants indicated with their mouse to whom they would like to throw the ball. In total, the ball had to be tossed 40 times. In the social exclusion condition, participants received the ball only three times during the first ten trials. Afterwards, the participant was completely excluded by both virtual players for the remainder of the game. In the inclusion condition, participants received the ball equally often as the other players.

2.3.5. Description of the ads. Participants were asked to write detailed descriptions about 5 printed advertisements of unknown beer brands. Participants first viewed each advertisement for 10 seconds and then had up to one minute to write a description. The order of the advertisements was randomized across participants. Stories were scored for need for affiliation, need for power and need for achievement. Need for affiliation scores were calculated to establish predictive validity of the PA-IAT measure and need for power and need for achievement scores were calculated to further establish discriminant validity of the PA-IAT. The descriptions of the ads were coded by two independent and trained scorers using Winter's Manual for Scoring Motive Imagery in Running Text (1994). Affiliation was scored whenever the description comprised excerpts that referred to (1) showing positive or intimate feelings towards other persons or nations, (2) sadness about separation or disruption of friendly relationships, or attempts to restore it, (3) engagements in affiliative, companionate activities, or 
(4) performance of a friendly, nurturant act. Power imagery was scored whenever a character in the story (1) engaged in forceful actions, (2) tried to control others, (3) attempted to influence, persuade, or convince others, (4) gave unsolicited help or advice, (5) tried to impress others, (6) mentioned his prestige or reputation, and (7) reacted emotionally to actions of others. Need for achievement was scored for (1) expressions of a standard of excellence, (2) descriptions of goals and performances in ways that suggest positive evaluations, (3) references to victories or competition with others without acts of aggression or power, (4) expressions of disappointment about failure or lack of excellence, or (5) mentioned unique accomplishment. Interrater reliability between the two scorers was good for all scored motives (Spearman-Brown coefficient: $r>.75$ ). Across the five ads, participants wrote on average 125 words $(S D=55.54)$, containing 2.96 need for affiliation excerpts $(S D=1.59), .15$ need for power excerpts $(S D=.38)$, and .48 need for achievement excerpts $(S D=.81)$. Because need for affiliation was positively correlated with the length of the written ad descriptions, the obtained score was corrected for the length of the description. For this, we regressed the raw score on length of the description and used the standardized residuals as dependent variable for our analyses (Smith, Feld, \& Franz, 1992). The scores of need for achievement and need for power were not correlated with the length of the written ad descriptions. Yet, for reasons of comparability, we applied the same correction to the raw need for power and need for achievement scores.

\section{Results}

The PA-IAT did not correlate with any explicit motive measures (see Table 1), suggesting that the implicit (PA-IAT) and explicit motive measures (PRF, CSS) are measures of different constructs. To test the predictive validity of the PA-IAT, we conducted a Generalized Linear 
Model with social exclusion (exclusion vs. inclusion) as a factor, the PA-IAT and PRF as continuous variables, and then number of affiliation related excerpts in the description of the ads as the to-be-predicted dependent measure. Results revealed that neither the main effects, nor the interaction between the PRF measure and social exclusion reached significance $(F s<2, p>$ .05).More importantly, we obtained a significant interaction between the PA-IAT measure of need for affiliation and social exclusion $\left(F(1,122)=6.45, p<.05, \eta^{2}=.06\right)$. For people who scored high on the PA-IAT measure of need for affiliation (1 SD above the mean), the number of affiliation related excerpts was higher when they were excluded than when they were not excluded during the Cyberball game $\left(M_{\text {inclusion }}=-.23, M_{\text {exclusion }}=.39, t(122)=2.49, p<.05\right)$. For people who scored low on the PA-IAT measure of need for affiliation (1 SD below the mean), social exclusion did not affect the number of affiliation related excerpts $\left(M_{\text {inclusion }}=.018\right.$, $\left.M_{\text {exclusion }}=-.29, t(122)=1.19, p>.05\right)$. Viewed differently, when socially excluded, the number of affiliation related excerpts increased as need for affiliation increased $(t(122)=2.40, p<.05)$. When not socially excluded, the PA-IAT measure of need for affiliation was not related to the number of affiliation related excerpts $(t(122)=1.06, p>.05)$. To further examine the discriminant validity of the PA-IAT, we repeated the analysis with the same predictors, but once with the number of need for achievement related excerpts and once with the number of need for power related excerpts as dependent variable. Results showed that none of the predictors reached significance in both models $(F s<2, p>.05)$.

\section{Discussion}

This study further explored the usefulness of the PA-IAT for the assessment of implicit motives. Recently, Slabbinck et al. (2011, in press) developed and validated a PA-IAT version 
for the assessment of need for power. Our results confirmed that the PA-IAT can be adapted to provide a valid measure of need for affiliation too. In line with implicit motivation theory (McClelland et al., 1989), we found that the need for affiliation PA-IAT did not correlate with explicit measures of need for affiliation. More crucially, it was predictive for non-declarative measures of implicit motives but only if need for affiliation was aroused.

The present study adds to our understanding of the functioning of implicit motives. Most importantly, our findings are consistent with the traditional implicit motive model of motivation (McClelland et al., 1989) in that our PA-IAT measure of need for affiliation was related to a nondeclarative index of need for affiliation only if this need was activated as the result of social exclusion. This pattern of results is completely in line with what would be expected for traditional motive measures such as the PSE. Consequently, the fact that different methods yield similar results can be considered as a further proof of the validity of the underlying psychological construct (i.e. implicit motives).More generally, having a PA-IAT measure in addition to the PSE measure allows one to verify core assumptions of implicit motives theories in a way that does not depend on idiosyncratic elements of one particular procedure.

Our results also have practical implications. First, compared to the content-coding techniques, the PA-IAT is much more economical to administer and to score. Consequently, the availability of such a measure could stimulate implicit motive research in general, and especially in areas where time efficient data collection is crucial. For example, if data collection involves field studies that require participation of hurried passengers, assessment of implicit motives by means of time consuming content-coding techniques might be too demanding and cause unacceptable drop-out rates. In fact, it is for exactly this reason that Kehr (2004) abandoned the PSE for the assessment of implicit motives. According to Kehr (2004), studies with management 
samples caused unacceptable drop-out rates on the PSE. Therefore, he decided to use the MultiMotive-Grid (Sokolowski, Schmalt,Langens, \& Puca, 2000) to assess implicit motives even though recent research puts the validity of grid techniques as measures of implicit motives into questions (Schultheiss et al., 2009). Perhaps, the PA-IAT can serve as a valid and time-efficient alternative to content-coding techniques. Second, the PA-IAT also has some clear advantages for preparing the data for analysis. Content-coding methods are labor-intensive. For instance, novice coders need at least 12 hours of scoring practice material before moving on to scoring any PSE protocols (Pang, 2010). In addition, coding of PSE stories is very time-consuming. A typical PSE study with 6 picture protocols from 100 participants will take between 20 and 50 hours to code (Pang, 2010). Contrary to content-coding techniques, the PA-IAT requires much less effort from researchers. Easy-to-use (PA-) IAT scripts are widely available and many standard procedures exist to convert raw (PA-) IAT data into analyzable motive scores (Greenwald, Nosek, \&Banaji, 2003). As such, the PA-IAT definitely lowers the entry barrier for novice implicit motive researchers and could stimulate implicit motive research.

Although the present study provides strong support for the validity of the PA-IAT as a measure of need for affiliation, more studies are required before the PA-IAT can truly be established as a valid alternative to the PSE. First, more replications are needed, preferably with diverse outcome variables and on diverse (multicultural) samples. For instance, our dependent variable focused on the relation between the implicit affiliation motive and memory processes. As such, we investigated cognitive processes that direct behavior rather than actual behavior. In line with Slabbinck et al. (2011, in press), our findings confirm that the PA-IAT is useful for the functional analysis of how implicit motives influence behavior, but it still needs to be investigated whether the PA-IAT is also able to predict actual motive relevant behavior such as 
attentional orientation to facial expressions (Schultheiss, 2008).Also, a growing body of evidence shows that the PSE measures of implicit motives are related to the release of hormones and brain activity(Hall, Stanton, \& Schultheiss, 2010). Thus, if the PA-IAT is a true measure of implicit motive, one would expect that the PA-IAT correlates with hormonal releases too. Unfortunately, validation of the PA-IAT with any biological markers is still lacking. Second, the study of Slabbinck et al. (in press) showed that the PA-IAT is only moderately related to the PSE. To figure out whether this moderate correlation is due to methodological or conceptual differences, a Multi-Trait-Multi-Method (MTMM) analysis is needed. However, as long as the PA-IAT was only available for need for power, such a MTMM analysis was not possible. Hence, with the construction of a need for affiliation PA-IAT, such a MTMM analysis is realistic. Third, it still needs to be examined whether the PA-IAT could be a useful measurement instrument for studying need for achievement. Fourth, both the PA-IAT measures of need for power and need for affiliation provide only a general assessment of the corresponding motives whereas many content-coding techniques can be used for the measurement of the different facets (e.g. facets of need for affiliation: intimacy, nurturance, ...) as well as the hope and fear components (e.g. need for affiliation as fear for rejection or hope of affiliation) of each motive (Winter, 1994). Finally, the specific benefits of pictorial stimuli in IAT measures of implicit motives need to be examined further. Our selection of pictorial stimuli relied upon motive-grid measures (Sokolowski et al., 2000). More specifically, to judge whether pictures were suitable for assessment of a particular implicit motive, participants rated the fit of a picture with descriptions of need for affiliation. However, as mentioned earlier, motive-grid measures are not convincingly validated as valid implicit motives measures (Schultheiss et al., 2009). Thus, it remains uncertain to what extent each picture we used in the PA-IAT represents implicit need for affiliation (and not the explicit 
need for affiliation). A more careful examination of the potential of a picture to represent an implicit motive could be the subject of future research. Regardless of these possible future developments, however, the present study already strongly suggests that the PA-IAT can provide a valid and useful measure of need for affiliation. 


\section{References}

Brunstein, J. C., \& Schmitt, C. H. (2004).Assessing individual differences in achievement motivation with the Implicit Association Test. Journal of Research in Personality, 38(6), $536-555$

De Houwer, J., Teige-Mocigemba, S., Spruyt, A., \& Moors, A. (2009). Implicit measures: A normative analysis and review. Psychological Bulletin, 135(3), 347-68.

Greenwald, A. G., McGhee, D. E., \& Schwartz, J. L. (1998). Measuring individual differences in implicit cognition: the implicit association test. Journal of Personality and Social Psychology, 74(6), 1464-80.

Greenwald, A. G., Nosek, B. A., \& Banaji, M. R. (2003). Understanding and using the Implicit Association Test: I. An improved scoring algorithm. Journal of Personality and Social Psychology, 85(2), 197-216.

Gschwendner, T., Hofmann, W., \& Schmitt, M. (2008). Differential stability: The effects of acute and chronic construct accessibility on the temporal stability of the Implicit Association Test. Journal of Individual Differences, 29(2), 70-79.

Hall, J., Stanton, S., \& Schultheiss, O. C. (2010). Biopsychological and neural processes of implicit motivation. In O. C. Schultheiss \& J. C. Brunstein (Eds.), Implicit motives (pp. 279307). New York, NY: Oxford University Press.

Jackson, D. N. (1984).Personality Research Form (3rd ed.). Port Huron: Sigma Assessment Systems.

Kehr, H. M. (2004). Implicit/explicit motive discrepancies and volitional depletion among managers. Personality and Social Psychology Bulletin, 30(3), 315-27. 
McClelland, D. C. (1985). How motives, skills, and values determine what people do. American Psychologist, 40(7), 812-825.

McClelland, D. C., Koestner, R., \& Weinberger, J. (1989). How do self-attributed and implicit motives differ? Psychological Review, 96(4), 690-702.

Pang, J. S. (2010). Content coding methods in implicit motive assessment: Standards of measurement and best practices for the picture story exercise. In O. C. Schultheiss \& J. Brunstein (Eds.), Implicit motives (pp. 119-150). New York: Oxford University Press.

Pickett, C. L., Gardner, W. L., \& Knowles, M. (2004). Getting a cue: the need to belong and enhanced sensitivity to social cues. Personality \&Social Psychology Bulletin, 30(9), 1095107.

Schultheiss, O. C. (2008). Implicit motives. In O. John, R. Robins, \& L. Pervin (Eds.), Handbook of Personality: Theory and Research (pp. 603-633). New York, NY: Guilford Press.

Schultheiss, O. C., \& Pang, J. S. (2007).Measuring implicit motives. Handbook of research methods in personality psychology (pp. 322-344).The Guilford Press.

Schultheiss, O. C., Yankova, D., Dirlikov, B., \& Schad, D. J. (2009). Are implicit and explicit motive measures statistically independent? A fair and balanced test using the picture story exercise and a cue- and response-matched questionnaire measure. Journal of Personality Assessment, 91(1), 72-81.

Sheldon, K. M., King, L. A., Houser-Marko, L., Osbaldiston, R., \& Gunz, A. (2007). Comparing IAT and TAT Measures of Power Versus Intimacy Motivation. European Journal of Personality, 21(3), 263-280.

Slabbinck, H., De Houwer, J., \& Van Kenhove, P. (2011). A pictorial attitude IAT as a measure of implicit motives. European Journal of Personality, 25(1), 76-86. 
Slabbinck, H., De Houwer, J., \& Van Kenhove, P. (in press.). Convergent, Discriminant, and Incremental Validity of the Pictorial Attitude Implicit Association Test and the Picture Story Exercise as Measures of the Implicit Power Motive. European Journal of Personality.

Smith, C. P. (1992). Motivation and personality: handbook of thematic content analysis (p. 708). New York: Cambridge University Press.

Smith, C. P., Feld, S. C.,\& Franz, C. E. (1992). Methodological considerations: Steps in research employing content analysis systems. In C. P. Smith (Ed.), Motivation and personality: Handbook of thematic content analysis (pp. 515-536). New York: Cambridge University Press.

Sokolowski, K., Schmalt, H. D., Langens, T. A., \& Puca, R. M. (2000).Assessing achievement, affiliation, and power motives all at once: The Multi-Motive Grid (MMG).Journal of Personality Assessment, 74(1), 126-145.

Williams, K. D. (2007). Ostracism. Annual Review of Psychology, 58, 425-52.

Winter, D. G. (1973).The power motive (p. 373). Free Press.

Winter, D. G. (1994). Manual for scoring motive imagery in running text (4th ed.). Ann Arbor, MI: University of Michigan. 


\section{Appendix}

The table below presents the items that were used to assess the fit of the pictures with need for affiliation. For each picture, reliability of the scale ratings was checked and the need for affiliation score was calculated as the mean score of the scale items.

This picture fits with someone who ...

... likes to spend time with others;

... likes to establish new bonds of friendship;

$\ldots$ is inclined to make concessions to others whose good-will is important to them;

... puts a lot of effort in maintaining and restoring positive relationships with others. 
Table 1. Correlations among motive measures.

1.2 .4$.

1. PA-IAT nAff

2. PRF nAff $\quad-.08$

3. CSS nAff $\quad-.03 \quad .40 * *$

4. CSS nPow $\quad .12 \quad-.10 \quad .14$

$\begin{array}{lllll}\text { 5. CSS nAch } & -.09 & -.13 & .09 & .27 * *\end{array}$

Note. PA-IAT $=$ Pictorial Attitude Implicit Association Test; PRF $=$ Personality Research

Form $;$ CSS $=$ Constant Sum Scale $;$ nPow $=$ Need for Power: $\mathrm{nAch}=$ Need for

Achievement; $\mathrm{nAff}=$ Need for Affiliation .

$* \mathrm{p}<.05 . * * \mathrm{p}<.01$ 